\title{
Pitfalls of Dynamic Contrast Enhanced MR Mammography (DCE-MRM) in Evaluation of Post-Biopsy Suspicious Breast Lesions
}

\author{
ENASS M. KHATTAB, M.D.*; TAREK M. SOBHY, M.D.* and ABD EL-HAFEZ M. EL-SHEWAIL, M.D.** \\ The Departments of Diagnostic Radiology* and General Surgery**, Faculty of Medicine, Zagazig University, Egypt
}

\begin{abstract}
Background: Breast MRI examination after diagnosis of breast cancer has been established to assess true extension of the disease. Therefore, it is important to evaluate any potential effect of biopsy on imaging characteristics.

Aim of the Study: This study aimed to evaluate efficacy of contrast enhanced magnetic resonance mammography (DCE-MRM) in evaluation of post-biopsy breast lesions.

Patients and Methods: Sixty-five female patients with suspicious breast lesions were included in this study. Dynamic contrast enhanced MR mammography (DCE-MRM) were performed to all patients pre and post biopsy, histopathological correlation was done as a golden slandered.

Results: There was over-estimation in 3 patients that were categorized as BIRADS3 based on pre-biopsy DCE-MRM and upgraded to BIRADS4 in post-biopsy examination, however their pathology were negative for malignant cells. There was under-estimation in 2 patients that were categorized as BIRADS5 based on pre-biopsy DCE-MRM and downgraded to BIRADS4 in post biopsy examination, their pathology were positive for malignant cells. Number of BIRAD4 cases increased in post-biopsy DCE-MRM by 5 lesions. The prebiopsy DCE-MRM accuracy was $91.8 \%$ and the post-biopsy was $85.7 \%$. $p$-value $<0.00001$ which was statistically highly significant.
\end{abstract}

Conclusion: Breast biopsy can cause uneven changes with possibility of alter the appearance on DCE-MRM. Changes in imaging characteristics can lead to inaccuracy of nature and extension of the tumor, which could have significant impact on treatment planning.

Key Words: Pre and post-biopsy dynamic contrast enhanced magnetic resonance mammography (DCE-MRM) - Breast biopsy-Sonomammography.

\section{Introduction}

DYNAMIC contrast enhanced MR mammography (DCE-MRM) is a good examination method in

Correspondence to: Dr. Enass M. Khattab The Department of Diagnostic Radiology Faculty of Medicine, Zagazig University, Egypt differentiation between malignant and benign breast lesions even in dense fibro-glandular parenchyma, it considers a promising tool in screening for breast lesions [1]

The total biopsy rates are higher in the suspicious breast lesions that include BI-RADS 3,4 \& 5 categorizes [2]. In the management of cases of early detected breast cancer breast conserving therapy (BCT) is taking the upper hand. So, it is so important to show the size, shape, extent and presence of other lesions before performing such strategies. Because of this, many surgeons ask for MRI before surgery even if biopsy was done [3] .

The breast biopsy can cause inflammatory changes with alternation of enhancement pattern and tumor size which will affect tumor appearance on MRI. These changes in imaging characteristics can lead to inaccuracy of nature and extension of the tumor, which could have significant impact on surgical and treatment planning with decrease in the diagnostic accuracy [3]

Breast biopsy can cause architectural changes, which will affect subsequent interpretive performance of DCE-MRM. A history of previous biopsy could also be associated with a higher DCE-MR mammography sensitivity because radiologists become more cautious to call an examination abnormal. Therefore, a prior breast biopsy may not only change subsequent interpretive sensitivity, it may also reduce specificity and increase the likelihood of a subsequent false-positive results [4]

Aim of the study: This study aimed to evaluate efficacy of contrast enhanced magnetic resonance mammography (DCE-MRM) in evaluation of postbiopsy breast lesions. 


\section{Patients and Methods}

This prospective study was performed on 65 female patients between the period from September 2016 - May 2017 at Zagazig University Hospitals. All cases had suspicious breast lesions (BI-RADS3, BI-RADS4 \& BI-RADS5) on basis of clinical examination in conjunction with mammography and US.

\section{Patient's inclusion criteria:}

- Female patients.

- Suspicious breast lesions (BI-RADS3, BI-RADS4 \& BI-RADS5) on basis of clinical, mammographic \& US examination.

- Patients with good renal functions.

\section{Patient's exclusion criteria:}

- Patients unwilling to complete the study.

- Male patients.

- Patients with renal impairment.

- Patients who have contraindications to do MRI (such as cardiac pace makers or cochlear implants).

\section{Imaging protocol:}

Dynamic contrast enhanced MR mammography (DCE-MRM) was performed on $1.5 \mathrm{~T}$ (Philips Achieva, class II a) by using standard bilateral dedicated breast coils. The imaging protocol consisted of axial, coronal, and sagittal images of both breasts.

Pre contrast $\mathrm{T} 1 \mathrm{~W}$ frame was acquired in the axial plane (FSE; -flip angle $=90$; $\mathrm{TR}=9.9 \mathrm{~ms}$; $\mathrm{TE}=$ 4.2ms; NEX, 1; 2-3mm slice thickness with no. gap; $512 \cdot 192$ matrix, $\mathrm{FVO}=35$ ). Acquisition of imaging started $10 \mathrm{~s}$ after the intravenous injection of $0.2 \mathrm{mmol} \mathrm{per} / \mathrm{kg}$ of gadopentetate dimeglumine (Gd-DTPA, Magnevist; flow rate $2 \mathrm{~mL} / \mathrm{s}$ ), imaging time with this frame was approximately $80 \mathrm{~s}$. Axial T1 WIs fat suppression and subtracted images after the DCE-series. A small region of interest (ROI) was placed selectively over the most intensely enhancing area of the lesion.

\section{Image inte rpretation:}

Two qualified radiologists with at least 10 years experience in breast MRI reading were sharing the cases (each radiologist evaluated same cases in pre and post-biopsy examination to avoid the interobserver variability). The morphologic and dynamic features were recorded and classified according to Fisher MRI findings which included 5 criteria: (1) Shape (Rounded, Oval, lobular, branching, specu- lated, stellate), (2) Margins of the enhanced lesion (Well defined, Indistinct/ill-defined), (3) Pattern of enhancement (Homogenous, non-enhancing internal septations, In-homogenous, ring enhancement), (4) Initial dynamic signal increase and (5) Post initial signal behavior according to the obtained time to signal intensity curve (5-7). Scores were given from 0 to 8 points and classified into five categories (category I: 0 points, negative and corresponded to BIRADS 1; category II: 1-2 points, benign and corresponded to BIRADS 2; category III: 3 points, probably benign and corresponded to BIRADS 3; category IV: 4-5 points, suspicious and corresponded to BIRADS 4; group V: 6-8 points, highly suspicious for malignancy and corresponded to BIRADS 5. Also we evaluate presence of multi-focality, multi-centricity, skin or lymph node involvement. After MRI examination, core biopsy was taken from all lesions, DCE-MRM was done again to 59 patients with abnormally enhanced lesions 28 days or more after biopsy for comparison.

\section{Statistical analysis:}

The sensitivity, specificity, accuracy, positive predictive value and negative predictive value of dynamic enhanced-MRM in the detection of pre\& post-biopsied suspicious breast lesions were calculated by using chi-square test.

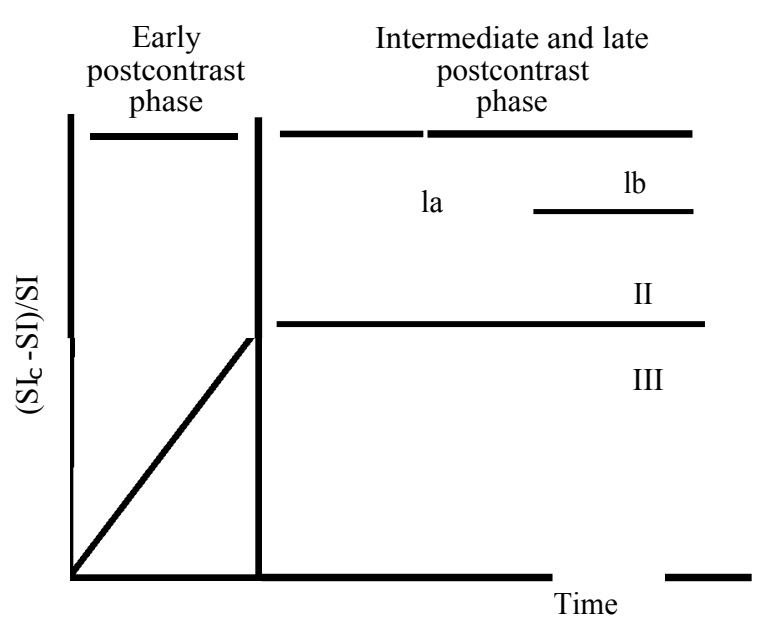

Fig. (1): Schematic drawing of the time-signal intensity curve types. Type I (Benign lesions) a straight (Ia) or curved (Ib) line; enhancement continues over the entire dynamic study. Type II (both benign and malignant lesions) is a plateau curve with a sharp bend after the initial upstroke. Type III (malignant lesions) is a washout time course $[6,7]$

\section{Results}

This study included 65 female patients with different suspicious breast lesions. Their ages ranged from 28 to 62 years with mean age 51.6 \pm 11.5 years (Table 1 ). Pre-biopsy soft tissue mam- 
mography, ultrasonography, conventional and dynamic contrast enhanced magnetic resonance mammography (DCE-MRM) examinations were performed to all the patients, post-biopsy DCE-MRM was performed to 59 patients whom showed previous enhancement, the results of the 65 patients were histopathologically correlated.

In mammography 34 patients (52.3\%) had focal masses. 16 patients $(24.6 \%)$ had focal asymmetrical density, architectural distortion was detected in 10 patients $(15.4 \%)$, other associated symptoms including skin thickening, nipple retraction, axillary lymphadenopathy and micro-calcifications as shown in Table (2). The ultrasonographic examinations of the 65 lesions showed; 38 patients $(58.5 \%)$ had solid masses, $10(15.4 \%)$ had complex lesions, 7 patients $(10.7 \%)$ had unilocular cyst, 10 (15.4\%) had localized abnormal asymmetrical parenchyma, other associated symptoms included skin thickening and diffuse breast edema, axillary lymph nodes enlargement, dilated ducts, and calcification inside the lesions as shown in Table (3).

Based on the sono-mammographic findings, 18 patients $(27.7 \%)$ were categorized as BI-RADS3, 26 patients $(40 \%)$ categorized as BI-RADS4 and 21 patients $(32.3 \%)$ categorized as BI-RADS5 (Fig. 2). Table (4) showed the histopathological results of the 65 lesions, we divided it into two groups: Group I included 23 patients (35.38\%) of benign lesions, fibroadenoma in 7 patients $(10.8 \%)$, periductal mastitis in 1 patient $(1.5 \%)$, fibrocystic changes in 5 patients $(7.7 \%)$, granulomatous chronic abscesses in 2 patients ( $3.1 \%$ ), inflammatory hyperplastic changes without atypia in 5 patients $(7.7 \%)$ and stromal fibrosis in 3 patients $(4.6 \%)$. Group II included 42 patients (64.62\%) had malignant lesions, they were subdivided into invasive ductal carcinoma in 22 patients $(33.8 \%)$, inflammatory carcinoma in 13 patients $(20 \%)$, invasive lobular carcinoma in 4 patients $(6.2 \%)$ and medullary carcinoma in 3 patients $(4.6 \%)$.

Table (5) showed enhancing pattern of the examined lesions, 6 lesions $(9.23 \%)$ showed no enhancement (biopsy revealed no malignant changes, they were 4 cases of fibrocystic disease, 2 cases of stromal fibrosis), 44 lesions $67.6 \%$ showed enhancing masses, 9 lesions (13.84\%) showed patchy regional (non-mass) enhancement and 6 lesions $(9.23 \%)$ showed enhancing foci and strands.

Table (6) showed pattern of the enhancing lesion, the pre-biopsy lesions were divided to; $30.5 \%$ with score (0) which represent (rounded, oval \& lobulated shapes), $69.5 \%$ with score (1) which represent (branching, spiculated \& stellate shapes), whereas the post-biopsy lesions divided to; $27.1 \%$ with score (0) \& $72.9 \%$ with score (1). As regards margin of enhancing lesion, the prebiopsy lesions were divided to; $44.1 \%$ with score (0) which represent (well defined margin) \& $55.9 \%$ with score (1) which represent (indistinct \& illdefined margin), on the other hand, post-biopsy lesions divided to; $37.3 \%$ with score (0) \& $62.7 \%$ with score (1). Considering the enhancement pattern, the pre-biopsy lesions were divided to; $55.9 \%$ with score $(0)$ which represent (homogenous enhancement \& non enhancing internal septations), $32.2 \%$ with score (1) which represent (inhomogeneous enhancement), $11.9 \%$ with score (2) which represent (ring enhancement), whereas post-biopsy lesions divided to; $49.2 \%$ with score (0), $40.7 \%$ with score (1), $10.2 \%$ with score (2). As regard the post-contrast initial signal intensity increase, the pre-biopsy lesions were divided to; $22.03 \%$ with score (0) which represent (less 50\%), 47.5\% with score (1) which represent (50\%-100\%), 30.5\% with score (2) which represent (>100\%), whereas post-biopsy lesions divided to; $13.6 \%$ with score (0), $54.2 \%$ with score (1), $32.2 \%$ with score (2). While signal intensity of the lesions 3-6 min after contrast injection in the pre-biopsy lesions were divided to; $15.3 \%$ with score (0) which represent (steady or continuous increase), $27.1 \%$ with score (1) which represent (plateau), 57.6\% with score (2) which represent (washout), and in post-biopsy lesions $6.8 \%$ with score $(0), 23.7 \%$ with score (1), $69.5 \%$ with score (2).

Table (7) showed type of time to signal intensity curve in the 59 enhanced lesions. Comparison between pre-biopsy and post-biopsy DCE-MRM grading of breast lesions in 59 patients were showed in table (8), while validity of the pre- and postbiopsy DCE-MRM was shown in Table (9).

Table (1): Age distribution of the 65 patients in this study.

\begin{tabular}{lcc}
\hline Age & Number & Percent (\%) \\
\hline $20-<30$ & 3 & 4.6 \\
$30-<40$ & 10 & 15.4 \\
$40-<50$ & 12 & 18.5 \\
$50-<60$ & 24 & 36.9 \\
$60-<70$ & 16 & 24.6 \\
\hline Total & 65 & 100 \\
\hline
\end{tabular}

The most common age group was $50-<60$ years (24 patients-36.9\%). 
Table (2): Mammographic imaging findings in the 65 lesions in this study.

\begin{tabular}{lcc}
\hline $\begin{array}{l}\text { Mammographic } \\
\text { findings }\end{array}$ & Number & Percent (\%) \\
\hline Masses & 34 & 52.31 \\
Asymmetries & 16 & 24.6 \\
Architectural distortion & 10 & 15.4 \\
Extremely dense breast & 5 & 7.7 \\
Associating findings: & & \\
$\quad$ Thickened Skin and & 13 & 20 \\
$\quad$ diffuse breast edema & & \\
$\quad$ Axillary lymphadenopathy & 16 & 24.6 \\
$\quad$ Nipple retraction & 16 & 24.6 \\
$\quad$ Micro-Calcification & 6 & 9.23 \\
\hline
\end{tabular}

Table (3): Ultrasound findings in 65 lesions in this study.

\begin{tabular}{lll}
\hline Lesions & Number & Percent \\
\hline Masses: & & \\
$\quad$ Solid & 38 & 58.5 \\
Complex & 10 & 15.4 \\
Cyst & 7 & 10.7 \\
Localized region of & 10 & 15.4 \\
abnormal asymmetrical & & \\
parenchymal echogenicity & & \\
Associated symptoms: & & \\
$\quad$ Axillary Lymphadenopathy & 23 & 35.4 \\
$\quad$ Dilated ducts & 8 & 12.3 \\
Thickened skin and edema & 13 & 20 \\
$\quad$ Micro-calcification (inside mass) & 4 & 6.2 \\
\hline
\end{tabular}

Table (4): Shows histopathological findings in the 65 lesions in this study.

\begin{tabular}{lcc}
\hline Lesion & Number & Percent (\%) \\
\hline Benign (23 patients): & 7 & 10.8 \\
$\quad$ Fibroadenoma & 1 & 1.5 \\
Periductal mastitis & 5 & 7.7 \\
Fibrocystic changes & 2 & 3.1 \\
Granulomatous chronic abscess & 5 & 7.7 \\
Inflammatory hyperplastic & & \\
$\quad$ changes without atypia & 3 & 4.6 \\
Stromal fibrosis & & \\
Malignant (42 lesions): & 22 & 33.8 \\
$\quad$ Invasive ductal carcinoma & 13 & 20 \\
Inflammatory carcinoma & 4 & 6.2 \\
Invasive lobular carcinoma & 3 & 4.6 \\
Medullary carcinoma & 65 & 100 \\
\hline Total & & \\
\hline
\end{tabular}

Table (5): Shows MRI enhancement patterns of (65) lesions in this study.

\begin{tabular}{lcc}
\hline Enhancement pattern & Number & Percent \\
\hline Nonenhancing lesions & 6 & 9.23 \\
Enhancing masses & 44 & 67.7 \\
Patchy regional enhancement & 9 & 13.84 \\
Enhancing foci and strands & 6 & 9.23 \\
\hline Total & 65 & 100 \\
\hline
\end{tabular}

Table (6): MRI criteria according to the Fischer system (5) evaluation scoring in 59 enhanced lesions.

\begin{tabular}{|c|c|c|c|c|c|}
\hline \multirow{2}{*}{ Criterion } & \multirow{2}{*}{ Points } & \multicolumn{2}{|c|}{ Pre-biopsy } & \multicolumn{2}{|c|}{ Post-biopsy } \\
\hline & & Number & Percent & Number & Percent \\
\hline \multicolumn{6}{|l|}{ 1- Shape of contrast enhanced lesions: } \\
\hline • Rounded, Oval, lobular & 0 & 18 & 30.5 & 16 & 27.1 \\
\hline - Branching, speculated, stellate & 1 & 41 & 69.5 & 43 & 72.9 \\
\hline \multicolumn{6}{|l|}{ 2- Margin of contrast enhanced lesion: } \\
\hline •Well defined & 0 & 26 & 44.1 & 22 & 37.3 \\
\hline • Ndistinct/ ill-defined. & 1 & 33 & 55.9 & 37 & 62.7 \\
\hline \multicolumn{6}{|l|}{ 3- Enhancing pattern of the lesion: } \\
\hline - Homogenous, non-enhancing internal septations & 0 & 33 & 55.9 & 29 & 49.2 \\
\hline •In-homogenous & 1 & 19 & 32.2 & 24 & 40.7 \\
\hline - Ring enhancement & 2 & 7 & 11.9 & 6 & 10.2 \\
\hline \multicolumn{6}{|l|}{ 4- Dynamic contrast enhancement pattern: } \\
\hline \multicolumn{6}{|l|}{ a- Initial signal intensity increase: } \\
\hline$\cdot$ Less than $50 \%$ & 0 & 13 & 22.03 & 8 & 13.6 \\
\hline$\cdot 50 \%$ to $100 \%$ & 1 & 28 & 47.5 & 32 & 54.2 \\
\hline - More than $100 \%$ & 2 & 18 & 30.5 & 19 & 32.2 \\
\hline \multicolumn{6}{|l|}{ b- Signal intensity at 3-6 min after contrast injection: } \\
\hline - Steady increase or continuous & 0 & 9 & 15.3 & 4 & 6.8 \\
\hline • Plateau & 1 & 16 & 27.1 & 14 & 23.7 \\
\hline -Washout & 2 & 34 & 57.6 & 41 & 69.5 \\
\hline
\end{tabular}


Table (7): Shows comparison between pre- \& post-biopsy findings of dynamic curve kinetics in the 59 lesions.

\begin{tabular}{lccccccc}
\hline & \multicolumn{3}{c}{ Prebiopsy } & & \multicolumn{3}{c}{ Postbiopsy } \\
\cline { 2 - 3 } Curve types & BIRAD3 & BIRAD4 & BIRAD5 & & BIRAD3 & BIRAD4 & BIRAD5 \\
\hline Type I & $8(72.7 \%)$ & $1(4.2 \%)$ & & & $3(37.5 \%)$ & $1(3.4 \%)$ & - \\
Type II & $3(27.3 \%)$ & $10(41.7 \%)$ & $3(12.5 \%)$ & & $5(62.5 \%)$ & $9(31.03 \%)$ & - \\
Type III & - & $13(54.2 \%)$ & $21(87.5 \%)$ & - & $19(65.5 \%)$ & $22(100 \%)$ \\
\hline Total & 11 & 24 & 24 & 8 & 29 & 22 \\
\hline
\end{tabular}

Table (8): Comparison between pre-biopsy and post-biopsy DCE-MRM in 59 breast lesions.

\begin{tabular}{|c|c|c|c|c|c|c|}
\hline \multirow{2}{*}{ Dynamic MRM } & \multicolumn{3}{|c|}{ Post-biopsy } & \multirow[b]{2}{*}{ Total } & \multirow{2}{*}{$X^{2}$} & \multirow[b]{2}{*}{$p$-value } \\
\hline & BIRAD3 & BIRAD4 & BIRAD5 & & & \\
\hline \multicolumn{7}{|l|}{ Pre-biopsy: } \\
\hline BIRADS3 & 6 & 4 & 1 & 11 & \multirow{3}{*}{31.9729} & \multirow{3}{*}{0.00001} \\
\hline BIRADS4 & 1 & 18 & 5 & 24 & & \\
\hline BIRADS5 & 1 & 7 & 16 & 24 & & \\
\hline Total & 8 & 29 & 22 & 59 & & \\
\hline
\end{tabular}

Table (9): Validity of the pre- \& post-biopsy DCE-MRM in the 59 lesions.

\begin{tabular}{lccccc}
\hline Dynamic MRM & Specificity (\%) & Sensitivity (\%) & PPV (\%) & NPV (\%) & Accuracy \\
\hline Pre-biopsy & 87.5 & 92.7 & 97.4 & 70 & $91.8 \%$ \\
Post-biopsy & 75 & 87.8 & 94.7 & 54.5 & $85.7 \%$ \\
\hline
\end{tabular}

Sonomammographic BI-RADS in 65 patients

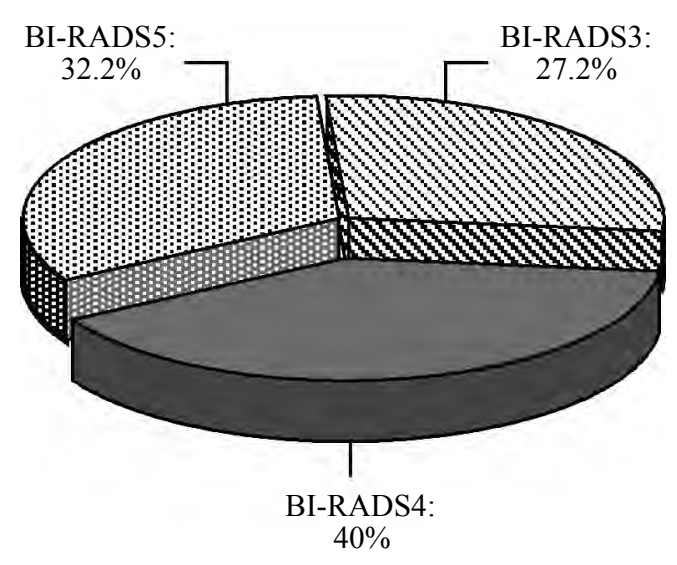

Fig. (2): Shows combined Sonomammographic BI-RADS classifications in 65 patients.

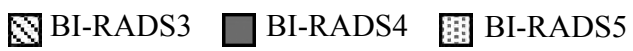



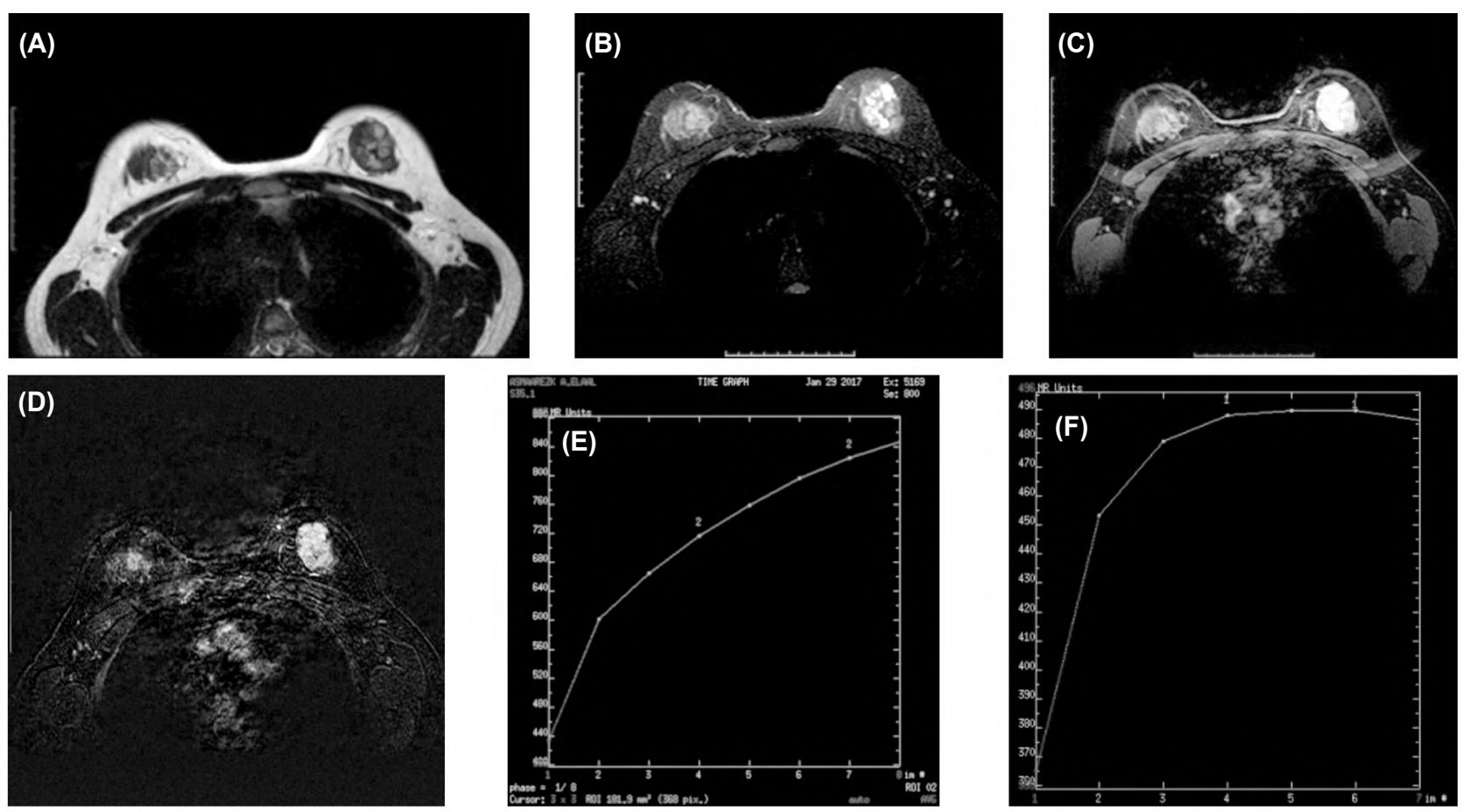

Fig. (3): A 38-year-old female patient with histopathologically proved left breast large fibroadenoma, it was primary categorized as BI-RADS3 and overestimated in in post biopsy exam to BI-RADS4, A \&B: Axial T2WI and STIR showing well defined lobulated hyperintense mass in upper outer quadrant of left breast. C \& D: Pre and post-biopsy axial T1FS after DCE-series showing intense homogeneous enhancement with non-enhancing internal septations. E: Pre-biopsy time to signal intensity type I curve (persistent) F: Post-biopsy time to signal intensity type II curve (Plateau).
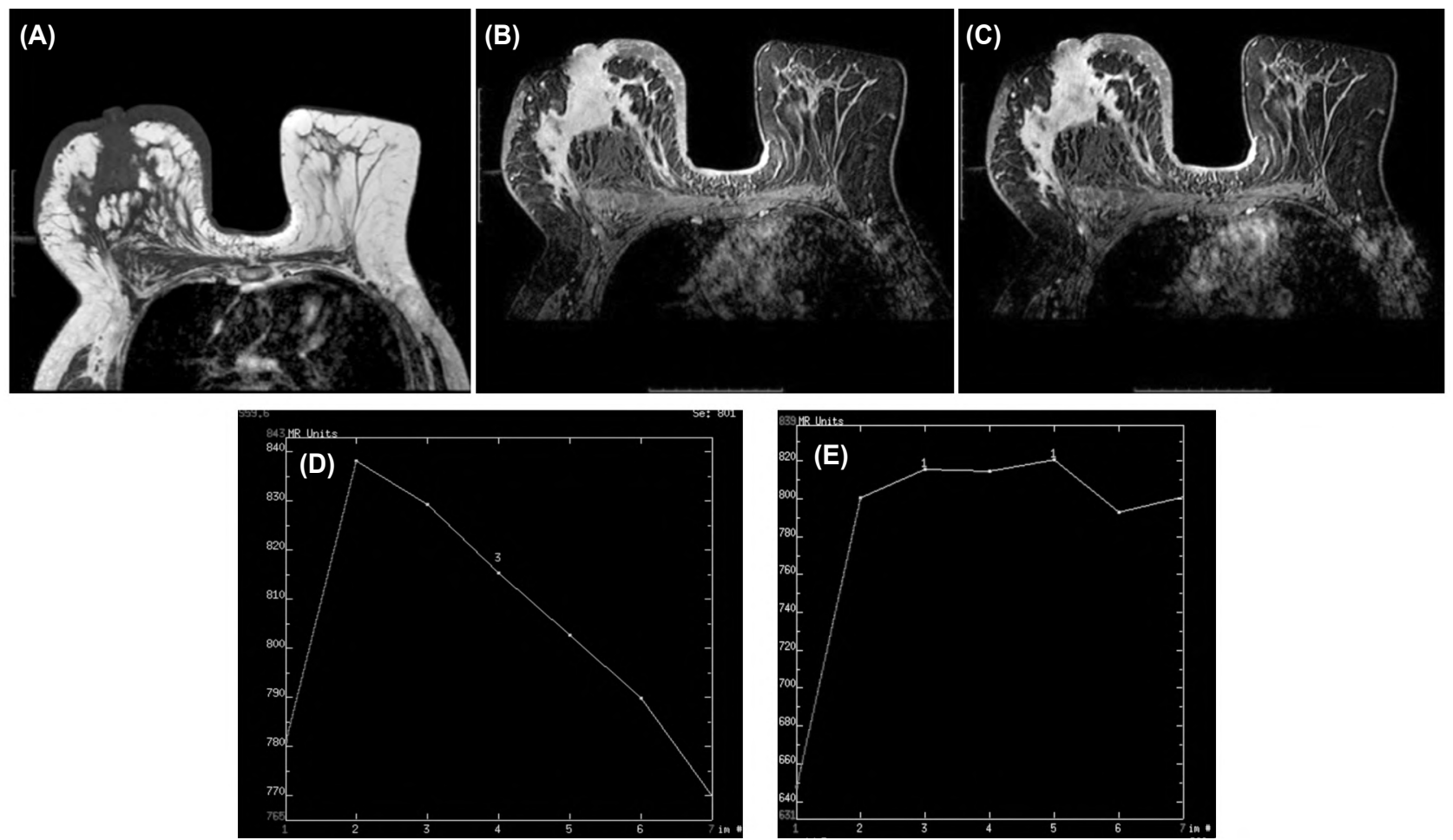

Fig. (4): A 54-year-old patient with RT breast diffuse tenderness and hardness (histopatologically proved inflammatory carcinoma) it was categorized as BI-RADS 5 in pre-biopsy exam and underestimated to BI-RADS4 in post-biopsy exam. A: Axial T2WI showing retroareolar indistinct heterogeneous signal intensity lesion with subcutaneous odema and skin thickening B \&C: Pre and post-biopsy axial T1FS after DCE-series showing retroareolar region of heterogeneous non-mass enhancement. D: Pre-biopsy time to signal intensity type III curve (rapid washout), E: Post-biopsy time to signal intensity type II curve (plateau). 

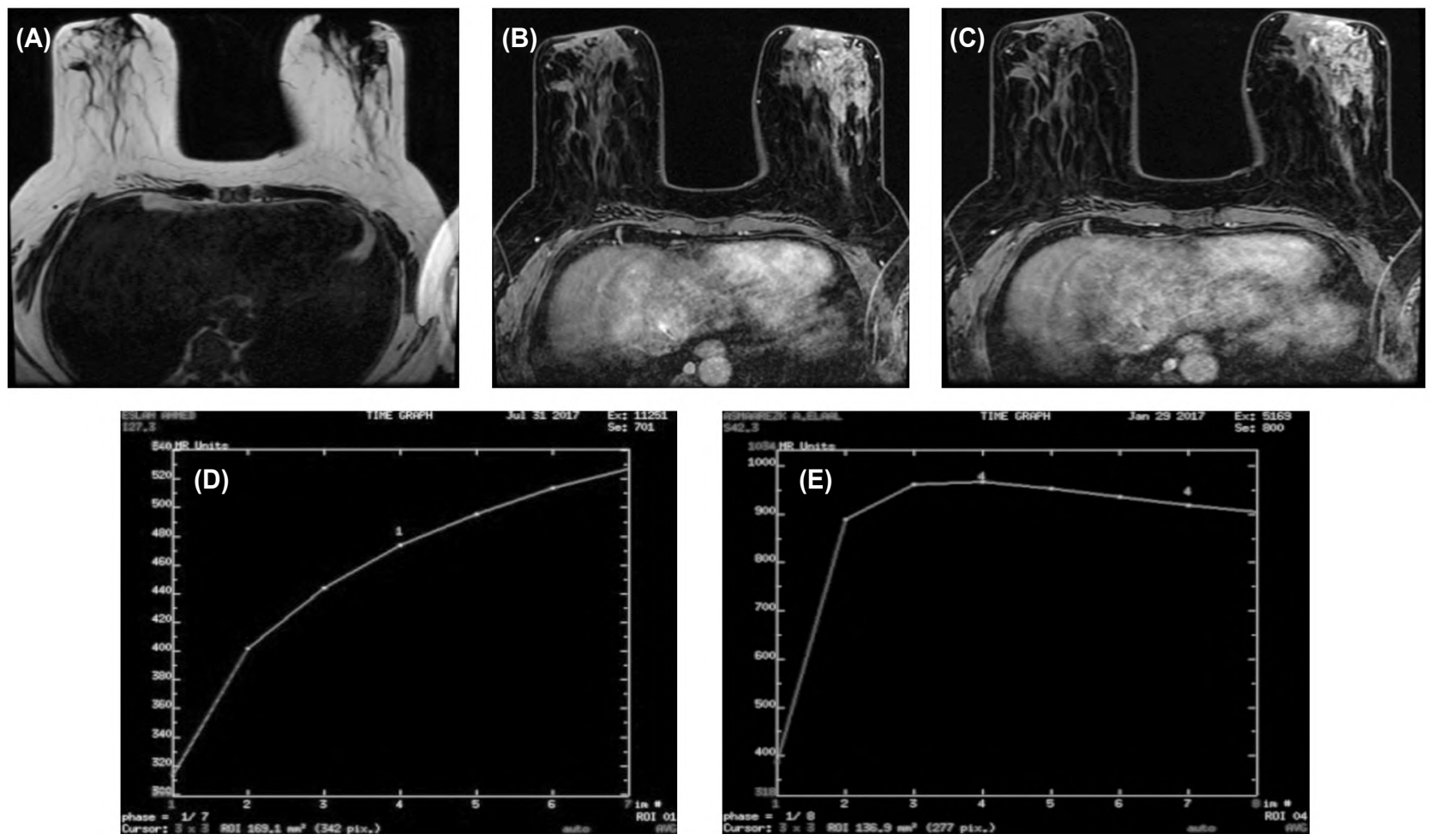

Fig. (5): A 49-year-old patient with Lt breast hardness (histopatologically proved localized inflammatory hyperplastic changes without atypia). It was primary categorized as BI-RADS 3 and overestimated in in post biopsy exam to BI-RADS4, A: Axial T2WI showing lower outer quadrant hypointense area, B \& C: Pre and post-biopsy axial T1FS after DCEseries showing intermediate heterogeneous regional enhancement in lower quadrant of Lt breast, D: Pre-biopsy time to signal intensity type I curve (persistent), and E: Post-biopsy time to signal intensity type II curve (plateau).
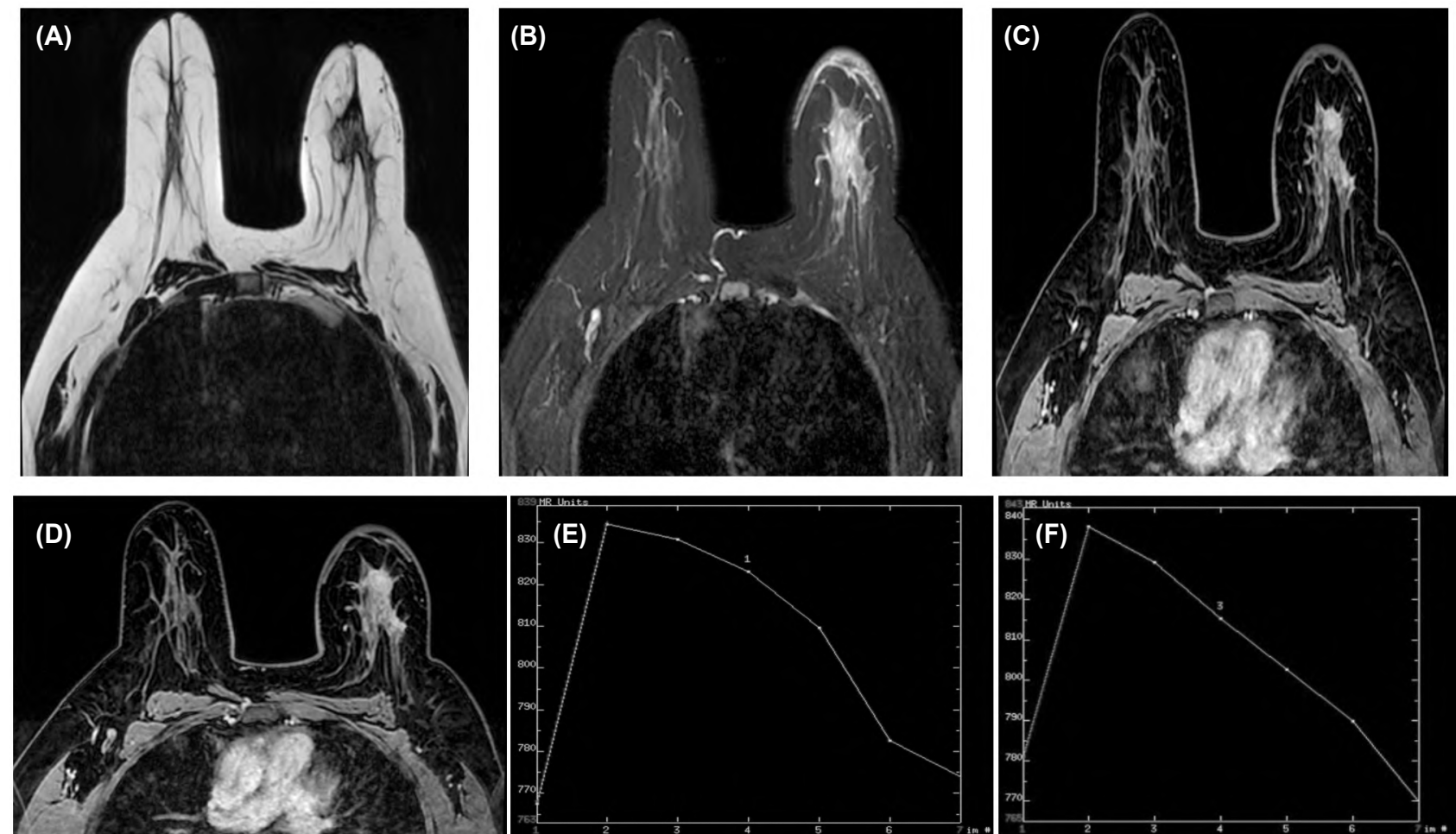

Fig. (6): A 58-year-old female patient with histopathologically proved left breast invasive ductal carcinoma, it was categorized as BI-RADS5 in pre and post-biopsy exam. A \&B: Axial T2WI and STIR showing large indistinct margin speculated hyperintense mass in left breast retroareolar region with mild skin thickening. C \& D: Pre and post-biopsy axial T1FS after DCE-series showing heterogeneous enhancement. E and F: Pre and post-biopsy time to signal intensity type III curve (rapid washout). 


\section{Discussion}

Breast biopsies are performed after 1\%-2\% of mammog $\neg$ raphy screenings when suspect areas on the mammograms cannot be explained by additional imaging. However, cancer is not detected in $65 \%$ $75 \%$ of these biopsies. Such high rates of biopsy demand that women and their health-care providers understand the adverse effects which affect the quality of subsequent Imaging modalities [4]

According to Macura et al., breast MR imaging should be performed after 28 days or more from any breast intervention. There is a positive relationship as the time interval between the intervention and MRI examination increase, a greatest influence on the specificity and NPV will be obtained, and increase progressively over time. Postintervention regions may be enhanced up to 6 months without radiotherapy and up to $18-24$ months after radiotherapy [8].

In the current study, we found that 12 out of 59 lesions $(18.5 \%)$ demonstrated changes in enhancement pattern after biopsy: 3 lesions (4.6\%) with homogenous washout kinetics became heterogeneous, 1 lesion (1.5\%) with ring enhancement washout became heterogeneous, 4 lesions $(6.2 \%)$ with progressive curve became washout, 3 lesions (4.6\%) with plateau became washout \& 1 lesion $(1.5 \%)$ with washout curve became plateau curve .This agreed with a study by Tirada et al., [3] who found that 8 out of 36 lesions (22\%) showed changes in enhancement pattern after biopsy: 3 lesions $(8.3 \%)$ with homogenous washout kinetics became heterogeneous, 3 lesions $(8.3 \%)$ with homogenous washout became persistent, and 1 lesion (2.8\%) with progressive curve became plateau.

Type 1 curve (persistent increase) was detected in this study in (72.7\%), (4.2\%) of BI-RADS 3 \& BI-RADS 4 respectively of pre-biopsy breast lesions on the other hand, it was detected in $(37.5 \%)$ \& $(3.4 \%)$ of BI-RAD3 \& BI-RADS4 respectively of post-biopsy breast lesions. The type II curve (plateau) was detected in $(27.3 \%),(41.7 \%) \&$ (12.5\%) of BI-RADS 3, BI-RADS4 \&BI-RADS5 respectively of pre-biopsy breast lesions and in $(62.5 \%) \&(31.03 \%)$ of BI-RAD3 \& BI-RADS4 respectively of post-biopsy breast lesions. The type III curve (washout) was detected in $(54.2 \%)$, (87.5\%) of BI-RADS 4 \& BI-RADS 5 respectively of pre-biopsy breast lesions and in $(65.5 \%) \&$ (100\%) of BI-RAD4 \& BI-RADS5 respectively of post-biopsy breast lesions.

There was over-estimation appeared in (3 cases) that were categorized as BI-RADS3 based on pre- biopsy DCE-MRM and upgraded by post-biopsy DCE-MRM to be categorized as BI-RADS4 however their pathology were negative for malignant cells. There was under-estimation appeared in (2cases) that were categorized as BI-RADS5 based on pre-biopsy DCE-MRM and downgraded in postbiopsy exam to be categorized as BI-RADS4, their pathology were positive for malignant cells. Number of BI-RADS4 cases increased in postbiopsy DCE-MRM by ( 5 cases) in comparison to the pre-biopsy examination. These findings were agreed with Liberman et al., [9], who stated that the overestimation of the BIRADS category in post-biopsy lesions is likely due to increased gadolinium enhancement following histological sampling which may obscure the margins of breast lesions, and affecting the interpretation of MRI images.

In the current study DCE-MRM was valuable diagnostic modality for evaluation of suspicious pre- and post-biopsy breast lesions. Sensitivity of pre-biopsy DCE-MRM in diagnosis of breast lesions was $92.7 \%$, specificity was $87.5 \%$, accuracy was $91.8 \%$, PPV was $97.4 \%$ and NPV was $70 \%$. Sensitivity of post-biopsy DCE-MRM was $87.8 \%$, specificity was $75 \%$, accuracy was $85.7 \%$, PPV was $94.7 \%$ and NPV was $54.5 \%$ (Table 8). And this agreed with Stephen et al. (4) who found a $2.7 \%$ reduced unadjusted sensitivity among women with a biopsy vs no biopsy (79.6\% vs $82.3 \%)$. They mentioned that interpretive performance in a biopsied woman appears to be different and less accurate than in an un-biopsied woman, a history of breast biopsy was associated with lower specificity, PPV, and accuracy.

A statistically significant difference between pre- and post- biopsy diagnosis of suspicious breast lesions by DCE-MRM was obtained in our study with $p$-value $=<0.00001$. While in a study done by Tirada et al., [3] they found that breast biopsy causes changes in enhancement pattern but does not significantly impact the accuracy of diagnosis or tumor size measurement.

In the present study MRI was done 28 days or more after breast biopsy, our results were statistically significant $p<0.00001$ (Table 9). And this agreed with a recent study done by Mennella et al., [10] whom used a cutoff value of 30 days as a time interval between biopsy and MRI, they concluded that the median absolute MRI-pathology difference was significantly higher in the group of patients in which the biopsy-MRI time interval was longer than 30 days $(p<0.05)$. 


\section{Conclusion:}

With a sensitivity and specificity of more than $70 \%$; DCE-MRM was a valid examination for the diagnosis of the suspicious pre- \& post- biopsy breast lesions. A statistically significant difference between imaging characteristics and diagnosis of pre- and post- biopsy DCE-MRM of the examined breast lesions $(p<0.0001)$ was obtained. Breast biopsy can cause biopsy-related inflammatory changes which may affect imaging characteristics and alter tumor appearance on MRI. These changes in imaging characteristics can lead to inaccuracy in the diagnosis with significant impact on surgical and treatment planning,

Recommendation: DCE-MRM is better to carried out before biopsy especially in lesions categorized as BIRADS3 and 4.

\section{Conflict of Interest:}

The authors declared that there is no potential conflicts of interest.

\section{References}

1- MÉNDEZ C.A., FERRARESE F.P., SUMMERS P., PETRALIA G., and MENEGAZ G.: DCE-MRI and DWI Integration for Breast Lesions Assessment and Heterogeneity Quantification. Int. J. Biomed. Imaging. Volume (2012), Article ID 676808, 8 pages, 2012.

2- SANGMA M., PANDA K., and DASIAH S.: A clinicopathological study on benign breast diseases. Journal of clinical and diagnostic research JCDR., 7 (3): 503, 2013.
3- TIRADA N., CHUKUS A. and KAPLAN S.S.: Does breast biopsy affect lesion enhancement characteristics or accuracy of tumor measurement on MRI? Radiological Society of North America 2013 Scientific Assembly and Annual Meeting, December 1- December 6, Chicago IL, 2013.

4- STEPHEN H., ABRAHAM B., GELLER M., et al.: Effect of Previous Breast Biopsy on the interpretive Performance of Subsequent Mammography. JNCI Oxford Journal, 102: $14,2010$.

5- BAUM F., FISCHER U., VOSSHENRICH R., et al.: Classification of hypervascularized lesions in CE MR imaging of the breast. Eur. Radiol., 12: 1087, 2002.

6- KUHL C.K., MIELCARECK P., KLASCHIK S., et al.: Dynamic breast MR imaging: Are signal intensity time course data useful for differential diagnosis of enhancing lesions? Radiology., 211:101-10, 1999.

7- KUHL C.K., and SCHILD H.H.: Dynamic image interpretation of MRI of the breast. J. Magn. Reson. Imaging., 12: $965-74,2000$

8- MACURA K.J., OUWERKERK R., JACOBS M.A. and BLUEMKE D.A.: Patterns of enhancement on breast MR images: interpretation and imaging pitfalls. Radiographics, 26 (6): 1719-1734, 2006

9- LIBERMAN L., MORRIS E.A., DERSHAW D.D., et al.; Ductal enhancement on MR imaging of the breast. Am. J. Roentgenol., 181: 519-525, 2003.

10- MENNELLA S., PAPARO F., REVELLI M., et al.: Magnetic resonance imaging of breast cancer: does the time interval between biopsy and MRI influence MRIpathology discordance in lesion sizing? Acta Radiologica, Vol. 58 (7) 800-808, 2017. 


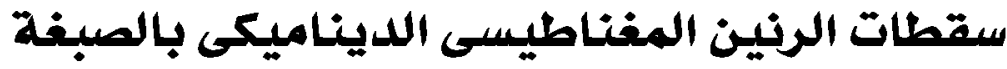

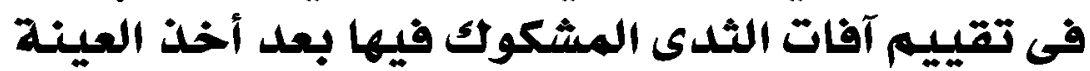

يعتبر تصوير أورام الثىى بالرنين المفناطيسى الديناميكى بالصبفة مكمل للتصوير بالماموجرام ويتميز بحساسيته الفائقة فى تشخيص سرطانات الثدى خاصة قى الثىى ذو كثافة انسجة عالية.

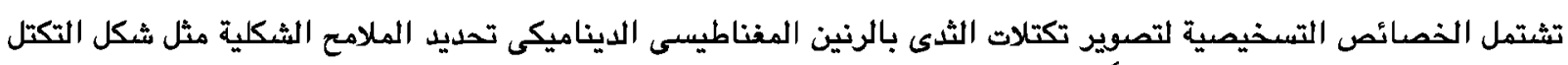

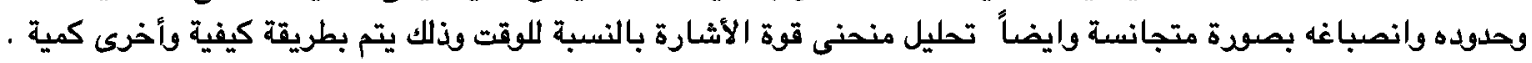

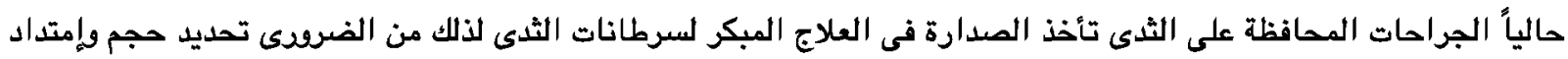

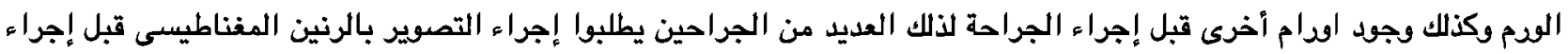
الجراحة حتى لو تم أخذ العينة.

تهدف هذه الدراسة لتحديد تأثير أخذ العينة فى قدرة الرنين المغناطيسى الديناميكى على تحديد خصائص أودام الثىى الكمية والكيفية.

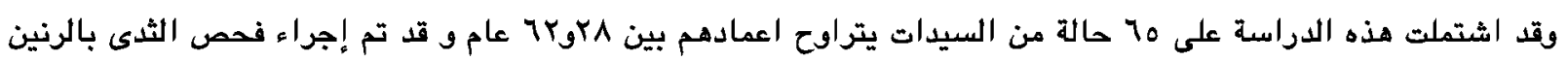

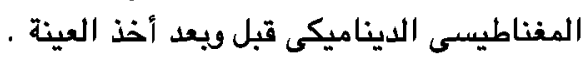

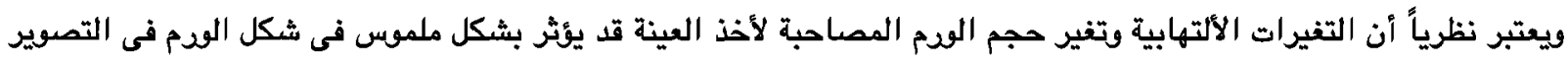

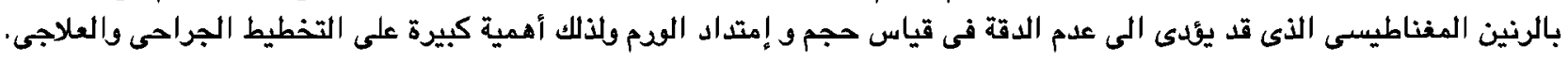

